\title{
Inactivation of one allele of the type II collagen gene alters the collagen network in murine articular cartilage and makes cartilage softer
}

M M Hyttinen, J Töyräs, T Lapveteläinen, J Lindblom, D J Prockop, S-W Li, M Arita, J S Jurvelin, H J Helminen

\begin{abstract}
Objective-To evaluate the influence of inactivation of one allele ("heterozygous knockout" or "heterozygous inactivation") of the type II procollagen gene (Col2a1) on the biomechanical properties and structure of the articular cartilage and subchondral bone in 15 month old mice.
\end{abstract}

Methods-Indentation stiffness of the humerus head articular cartilage was measured by a microindentation method. Cartilage and subchondral bone were prepared for digital densitometry of proteoglycans (PGs), polarised light microscopy (PLM) of collagen, and osteoarthrosis (OA) grading.

Results-Heterozygous inactivation of the Col2a1 gene softened articular cartilage $(p=0.002)$ as measured by indentation stiffness ((mean (SEM) 0.50 (0.07) MPa $v$ $0.94(0.13) \mathrm{MPa}$ in controls). Fibrillar collagen network exhibited lower birefringence in the intermediate $(p=0.04)$ and deep zones $(p=0.01)$ of cartilage by PLM, indicating either decreased collagen content or a lower degree of fibril parallelism

Department of Anatomy, University of Kuopio, Finland

M M Hyttinen

T Lapveteläinen

$\mathrm{J}$ Lindblom

H J Helminen

Department of Clinical Physiology and Nuclear Medicine, Kuopio University Hospital, Finland J Töyräs

J S Jurvelin

Centre for Gene Therapy, School of Medicine, MCP Hahnemann University of Health Sciences, Philadelphia, PA, USA

D J Prockop

S-W Li

M Arita

Correspondence to: Dr H J Helminen,

Department of Anatomy,

University of Kuopio, PO

Box 1627, FIN-70211

Kuopio, Finland

Heikki.Helminen@uku.fi

Accepted 12 July 2000 cally, articular cartilage can be regarded as a hierarchically organised material, in which collagen contributes to tensile properties and withstands shear forces, whereas proteoglycans are primarily responsible for the viscous osmotic resistance during compression. ${ }^{56}$ The stabilising and linking roles of the noncollagenous matrix proteins are poorly understood. Interestingly, the cartilage matrix protein family (matrilins) shows affinity to both aggrecan and collagen.

The three dimensional collagen network of articular cartilage is thought to be crucial for the mechanical properties of matrix during joint loading and cartilage deformation. ${ }^{8}$ In some forms of hereditary chondrodysplasias the formation of type II procollagen can be disturbed owing to single base mutations, splicing defects, deletions, insertions, or duplications. ${ }^{9}$ Depending on the type of gene defect, the osteoarthritic phenotype can vary from mild to severe. The extracellular matrix of articular cartilage can accommodate large local strains without losing its integrity. ${ }^{10}$ The superficial collagen network is principally formed by fibrils oriented parallel with the surface. Fibrils bend down into the intermediate zone to run at right angles to the joint surface in the deep zone and calcified cartilage. ${ }^{11-14}$ In the different zones, collagen fibrils probably respond differently to static and dynamic loading. ${ }^{15}$ Organisation of the collagen network varies between different joints and cartilage surfaces. ${ }^{16}$ Differences in the thickness of the cartilage zones containing collagen fibrils that are parallel, oblique, or perpendicular to the cartilage surface, seem to contribute to the biomechanical properties of articular cartilage. ${ }^{17}$ Remarkable variability in collagen organisation can be found in the same joint areas in different species, especially in the superficial zone. ${ }^{18}$

In osteoarthrosis (OA), early degeneration of articular cartilage by superficial fibrillation and clefting of the tissue leads gradually to irreversible damage and impaired function of the whole joint organ: articular cartilage, subchondral bone, ligaments, and the synovium. It is not clear what is the "point of no return" in progressive degeneration. Also, it is not known whether changes occur first in PGs or matrix collagens and which events trigger the final degradative process. One possibility is that the breakdown of the collagen fibril network marks the "point of no return". ${ }^{19}$ The metalloproteinase mediated matrix degeneration pathway is universal irrespective of the primary or secondby the covalently linked type IX and XI collagens $^{1-3}$ and possibly also by proteoglycans (PGs) - for example, decorin. ${ }^{4}$ Biomechani- 
ary origin of the disease-that is, idiopathic or induced OA. In an early stage of the disease, breakdown and denaturation of the superficial type II collagen fibrils can be shown by immunocytochemistry. ${ }^{19}$ Additional data supporting early derangement of the superficial collagen fibrils have been reported after long distance running ${ }^{16}$ or altered load bearing after valgus osteotomy. ${ }^{20}$ Therefore, it appears that protection and integrity of the superficial collagen network are imperative for the prevention of irreversible matrix degeneration. ${ }^{21}$

Several reports have shown that Col2al gene mutations can cause the development of OA and mild to severe chondrodysplasias. ${ }^{3}{ }^{22}$ Data from transgenic mouse experiments have further confirmed that normal fibril formation of type II collagen is disturbed if mutated pro$\alpha 1$ (II) chains are synthesised. ${ }^{23}{ }^{24}$ To date, however, the effects of the reduced formation of normal type II collagen fibrils on the development of adult murine cartilage and bone properties are not known.

Here we describe the structural and biomechanical properties of adult articular cartilage and subchondral bone of humerus head in transgenic mice with inactivation of one allele of the murine Col2al gene coding for type II procollagen. The biomechanical properties of cartilage were assessed by indentation measurements. The birefringent collagen network architecture of the articular cartilage and subchondral bone was analysed with quantitative polarised light microscopy (PLM). PGs of articular cartilage were measured by staining with safranin $\mathrm{O}$ and digital densitometry.

\section{Materials and methods}

ANIMAL EXPERIMENTATION

An inbred C57BL/6 mouse line having heterozygous inactivation of one allele of the Col2a 1 gene was used. This heterozygous knockout reduced the synthesis levels of pro- $\alpha 1$ (II) chains to about half the normal level, ${ }^{25}$ but no gross disease developed in cartilaginous tissues. ${ }^{24}$ The genotype of newborn mice was verified by a polymerase chain reaction as earlier described. ${ }^{26}$ Littermates with two wild-type alleles of the Col2a1 gene served as controls. After weaning, knockout $(n=15)$ and control $(n=19)$ male mice were housed under standardised conditions in individual cages having a surface area of $0.24 \mathrm{~m} \times 0.42 \mathrm{~m}$. At the age of 15 months, the heterozygous knockout mice weighed 39.5 (1.3) g (mean (SEM)) and the controls 38.7 (1.4) g. Water and R36 food pellets (Lactamin, Sweden) were given freely. At the age of 15 months, the mice were killed with an overdose of Avertin. ${ }^{27}$ The Animal Care and Use Committee of the University of Kuopio approved the design of the experiment.

\section{BIOMECHANICAL MEASUREMENTS}

The proximal end of the humerus was dissected free, immediately frozen in liquid nitrogen, and stored at $-80^{\circ} \mathrm{C}$. After blind coding and randomisation the specimens were thawed and the cartilaginous bone ends, including $1 \mathrm{~cm}$ bone shaft, were mounted on a three dimensional sample holder with thermal wax and immersed in phosphate buffered saline. The biomechanical measurements were performed with a custom made, computer controlled system. ${ }^{28} \mathrm{~A}$ cylindrical, impervious, plane-ended indenter (diameter $0.350 \mathrm{~mm}$, pre-stress $2.0 \mathrm{kPa}$ ) was positioned perpendicular to the articular surface under stereomicroscopic control (fig 1A). Mechanical measurements were carried out with a stepwise stress-relaxation indentation technique to obtain a typical strain of $20 \%$ of cartilage thickness. After every $3 \mu \mathrm{m}$ step the cartilage was relaxed to equilibrium for $200 \mathrm{~s}$ under the prevailing strain (fig 1B). During relaxation, stress and strain values were obtained, and indentation stiffness was determined from the slope of the equilibrium stress-strain curve (fig 1C). Cartilage thickness was determined by pushing a thin needle through the cartilage with the material testing device and simultaneously measuring the force and position..$^{29} 30$ The location of the cartilage surface and cartilagebone interface was recorded from the sharp changes in the force-position curve and from these data cartilage thickness was determined. The biomechanical measuring system consisted of a $250 \mathrm{~g}$ load cell (resolution $0.01 \mathrm{~g}$ ) (Sensotec, Columbus, OH, USA), a precision motion controller PM500-C with 1A actuator (resolution $0.1 \mu \mathrm{m}$ ) (Newport, Irvine, CA, USA), and LabView control software (National Instruments, Austin, TX, USA). For precise cutting of histological sections, the site of the indentation measurement was marked with a drop of ink under a stereomicroscope.

PROCESSING OF SPECIMENS FOR MICROSCOPY The proximal humerus ends were fixed in neutral phosphate buffered $10 \%$ formalin, $\mathrm{pH} 7.4$, decalcified in $10 \%$ formalin-EDTA at $18^{\circ} \mathrm{C}$ for 14 days, dehydrated in ascending alcohols, and embedded in Paraplast PlusR (Sherwood Medical, St Louis, MO, USA). Successive $3 \mu \mathrm{m}$ thick sections were cut with an LKB Historange rotary microtome (LKB, Bromma, Sweden) for safranin O staining of PGs, and $5 \mu \mathrm{m}$ thick sections for PLM of collagen. After dewaxing in xylene and rehydration in graded alcohols, the sections were processed for PLM $^{1631}$ or digital densitometry of PGs as previously described. ${ }^{32}$ Finally, the sections were mounted with DePeX mounting medium (BDH, Poole, UK).

POLARISED LIGHT MICROSCOPY OF COLLAGEN IN ARTICULAR CARTILAGE AND SUBCHONDRAL BONE PLM analysis of collagen network was made from unstained sections according to the principles described by Arokoski et al. ${ }^{1631}$ The sections were examined with a Leitz Ortholux II microscope equipped with crossed polariser and analyser, strain-free optics, a 16x/0.45 NA Fluotar objective (Leitz, Wetzlar, Germany), and an interference monochromator $(\lambda=595$ (10) nm, Optometrics Inc, Ayer, MA, USA). A squared pixel side corresponded to $1.43 \mu \mathrm{m}$. 12-Bit greyscale images were captured with a Photometrics $\mathrm{CH} 250$ cooled digital camera (Photometrics Inc, Tucson, AZ, USA) and converted to optical retardation images with 
A
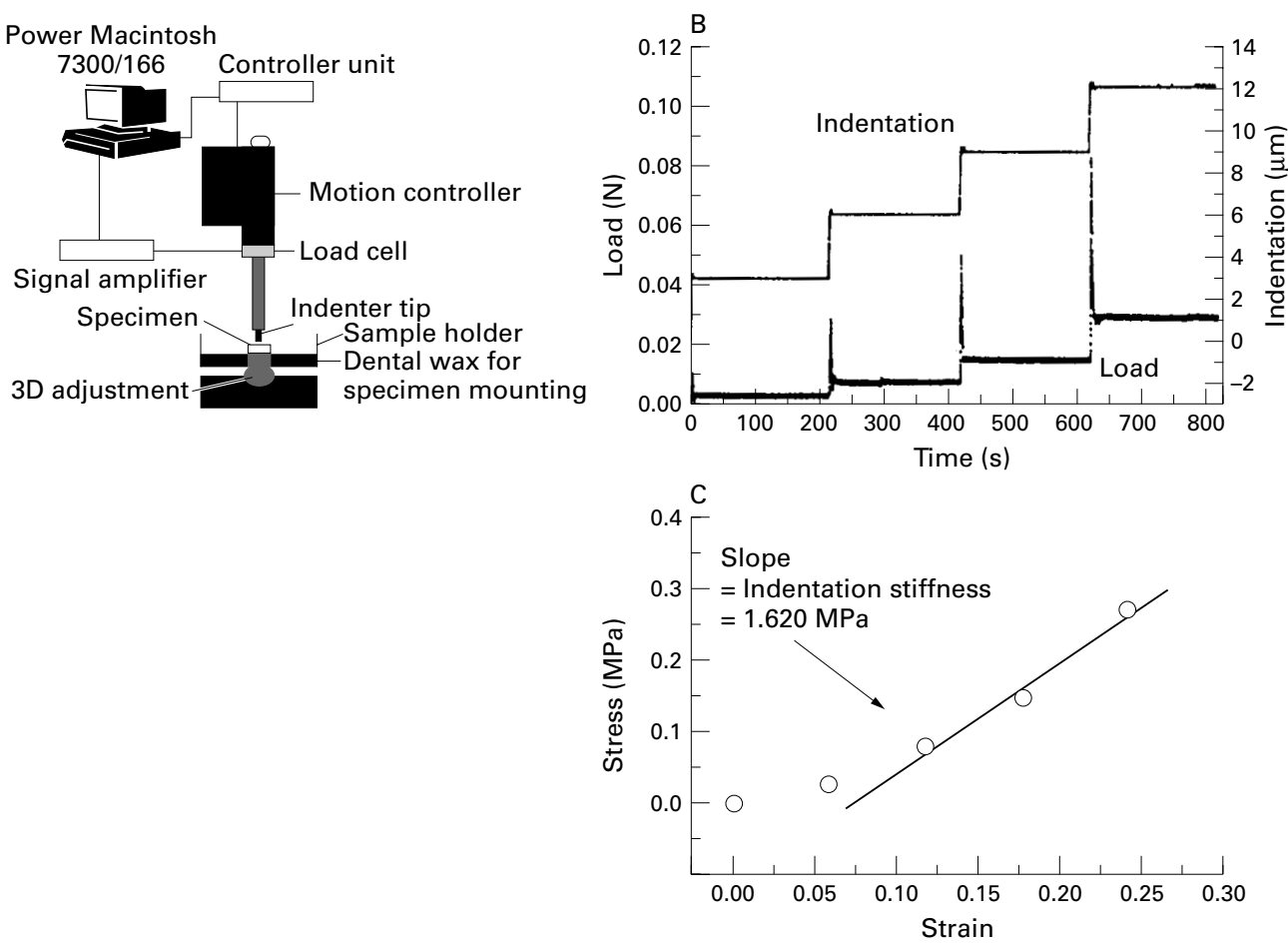

Figure 1 (A) Schematic presentation for the indentation measurement setup. (B) A typical series of four consecutive indentation stress relaxation steps ( $3 \mu \mathrm{m}$ for each step with ramping speed of $1 \mu \mathrm{m} / \mathrm{s}$ ) causing a total strain of $24 \%$ in mouse humerus articular cartilage (full thickness about $50 \mu \mathrm{m}$ ). After 200 s of relaxation the next step was taken. (C) Equilibrium stress-strain curve and determination of indentation stiffness from the stress-strain slope.

IPLab software (Signal Analytics, Vienna, VA, USA) according to the inverse Fresnel equation. ${ }^{16}$ Cartilage surface was positioned parallel to the analyser plane of the microscope and a perpendicular, $250 \mu \mathrm{m}$ wide rectangular region of interest was delineated at the same site at which biomechanical measurements were made. Because the signal intensity of collagen under linearly polarised light depends on fibril direction, linearly polarised light was used for determination of the borders of the collagen fibrils oriented (a) parallel, (b) obliquely, and (c) perpendicularly to the cartilage surface as previously described. ${ }^{16}$ Histologically, the fibrils were located in the superficial, intermediate, and deep cartilage zones, respectively. The whole cartilage and subchondral bone thicknesses were measured and the upper and lower margins of the subchondral plate were registered. Area integrated retardation was used as an estimator of the birefringent collagen fibril parallelism and content. ${ }^{16}$ Area integrated retardation measurements were made from semicircularly polarised light microscope images, also, to show fibrils situated in the direc-

Table 1 Osteoarthrosis severity and prevalence after inactivation ("knockout") of one allele of the Col2a1 gene

\begin{tabular}{|c|c|c|c|c|}
\hline Group & $\begin{array}{l}\text { Grade } 0 \\
(\%)\end{array}$ & $\begin{array}{l}\text { Grade } 1 \\
(\%)\end{array}$ & $\begin{array}{l}\text { Grade } 2 \\
(\%)\end{array}$ & $\begin{array}{l}\text { Grades } 3 \text { and } 4 \\
(\%)\end{array}$ \\
\hline Heterozygous Col2a1 knockouts $(n=15)$ & 26.7 & 66.7 & 6.6 & 0.0 \\
\hline Control mice $(n=19)$ & 78.9 & 21.1 & 0.0 & 0.0 \\
\hline
\end{tabular}

${ }^{\star}$ Comparison of healthy (grade 0 ) and diseased (pooled grades from 1 to 4 ) OA scores between knockout and control mice by $\chi^{2}$ statistics.

Grades: $0=$ intact articular cartilage; $1=$ superficial lesions not affecting the intermediate zone; 2 $=$ lesions extending to the intermediate zone; 3 and $4=$ lesions reaching the deep cartilage and subchondral bone, respectively. tion of extinction (at 45 degrees) in relation to the polariser and analyser. This took place as follows: the original, linearly polarised image was combined with another image of the specimen taken after parallel rotation of both polariser and analyser by 45 degrees. The position of the specimen was not changed between the exposures. The two images were combined with Boolean maximum function with the IPLab software. This generated the semicircularly polarised light image for measurements. The cartilage zones $(a)$ to $(c)$ and subchondral bone borders were transferred to the semicircularly polarised light image, and for each mouse the zone thickness weighted area integrated retardation values were determined for zones $(a)$ to $(c)$ as well as for subchondral bone. ${ }^{16}$ The volume fraction of subchondral bone was estimated from semicircularly polarised light images by binarising the bone structures using a constant threshold value. Based on the preferential collagen orientation assessed with linearly polarised light, the average cartilage thickness and superficial, intermediate, and deep zone thicknesses were calculated from control and knockout mice.

DIGITAL DENSITOMETRY OF PROTEOGLYCANS Safranin O distribution ${ }^{32}$ was analysed with digital densitometry ${ }^{20}$ using the same camera, microscope adjusted to standard transmission mode, optics, and IPLab software as in PLM. The system was calibrated with a neutral density filter set (Schott, Mainz, Germany) between 0 and 3.6 absorbance units. Stain absorbance was determined at $492 \mathrm{~nm}$ monochromatic light in 20 successive zones from the 


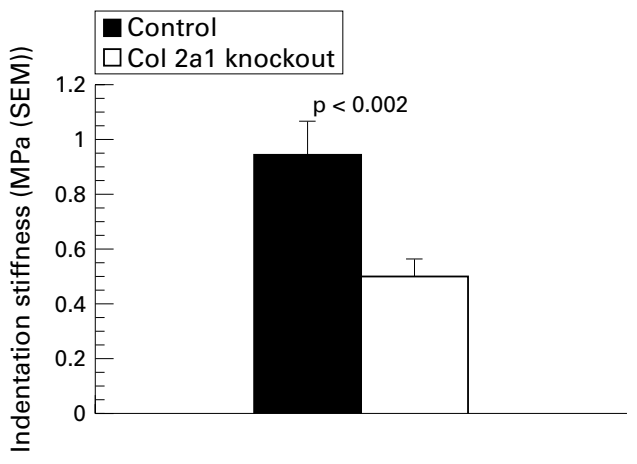

Figure 2 Indentation stiffness of articular cartilage (mean (SEM)) in the head of the humerus of the herterozygous Col2a1 knockout and control mice.

surface to the osteochondral junction. For each mouse, zone thickness weighted, area integrated optical density (that is, absorbance) was calculated for superficial, intermediate, and deep zones according to the thickness division obtained by the PLM.

GRADING OF OSTEOARTHROTIC LESIONS

The incidence and severity of osteoarthrotic lesions were graded from $3 \mu \mathrm{m}$ thick safranin $\mathrm{O}$ stained sections. A scoring system previously used to grade murine OA was adopted..$^{24}$ Table 1 explains the grading. Five successive safranin $\mathrm{O}$ stained sections were examined and the mode values of OA grades were calculated.

STATISTICAL METHODS

Data comparisons between the knockout and control groups were made with the nonparametric Mann-Whitney U test. Indentation stiffness, pooled PG content, cartilage thickness, pooled optical retardation of collagen, subchondral bone volume fraction, and optical retardation were included in a linear factorial correlation model in which indentation stiffness was dependent on these five factors. Standard error of the mean (SEM) was used as an estimator of variation.

OA prevalence was tested with $\chi^{2}$ statistics. OA scores 1 to 4 were pooled as "diseased" and grade 0 represented "healthy". Observed frequencies in knockout mice were compared with the frequencies expected, obtained from the control group.

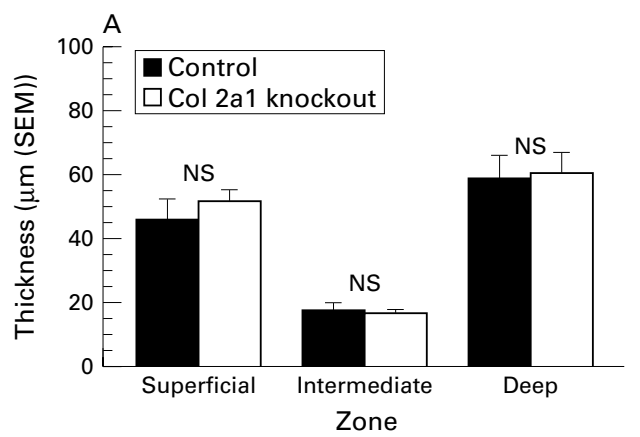

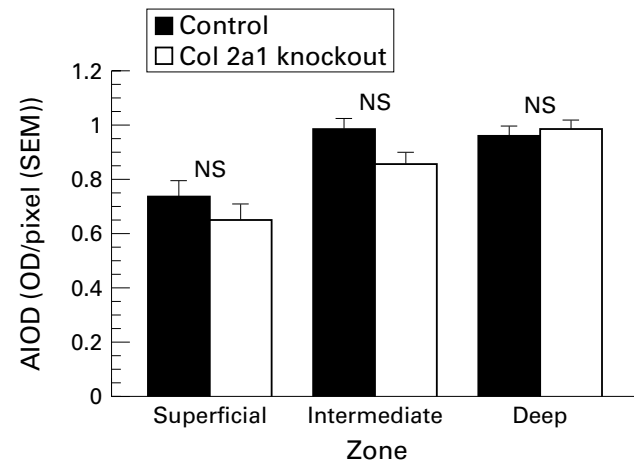

Figure 4 Safranin $O$ absorbance expressed as area integrated optical density (AIOD) in superficial, intermediate, and deep zones of articular cartilage. Zones are the same as those in figs $3 A$ and B. Mann-Whitney $U$ test.

\section{Results}

In histological sections, no qualitative differences in articular cartilage or subchondral bone morphology were seen between control mice and mice with heterozygous inactivation of the Col2al gene. However, there was a statistically significant increase in OA prevalence: $73 \%$ of the knockout mice but only $21 \%$ of the control mice showed signs of cartilage degeneration $(p=0.002)$ (table 1$)$. Most of the OA lesions were superficial and none of them extended into the deep zone of articular cartilage. In knockout mice the articular cartilage was significantly softer; the indentation stiffness was only about $53 \%$ of that in control mice $(\mathrm{p}=0.002)$ (fig 2$)$.

The heterozygous Col2a1 gene knockout did not change the total thickness of articular cartilage nor the thickness of the zones containing the collagen fibrils oriented tangentially, obliquely, or perpendicularly to the surface (fig 3A). However, in the intermediate and deep zones of the knockout mice, the area integrated retardation of polarised light due to collagen was about $78 \%$ and $80 \%$ (intermediate and deep, respectively) of the control mice birefringence $(\mathrm{p}<0.05-0.01)$ (fig 3B). When the zonal division obtained by the PLM measurements of the major course of collagen fibrils was used, the PG distribution in knockout mice did not differ from that of the control mice in any of the three zones as assayed by safranin $\mathrm{O}$ staining. However, the values seemed to be lower in the

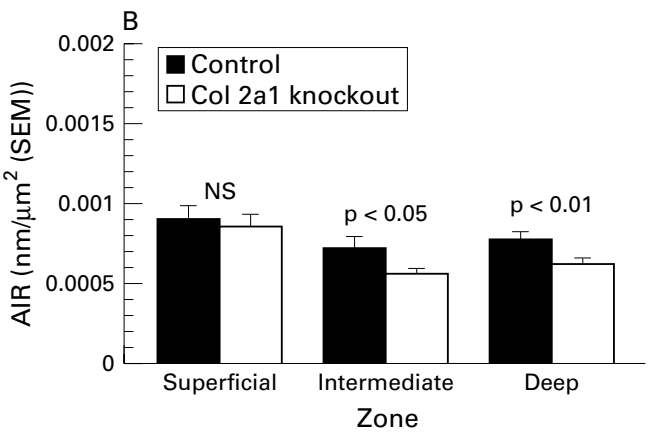

Figure 3 (A) Thickness (mean (SEM)) of superficial, intermediate, and deep zones in control and knockout mice. Absolute cartilage thickness is the sum of these three zones. Mann-Whitney $U$ test. (B) Collagen birefringence expressed as area integrated retardation (AIR, mean (SEM)) of semicircularly polarised light in the superficial, intermediate, and deep zones of articular cartilage of heterozygous Col2a1 knockout and control mice. The three zones contain collagen fibrils oriented tangentially, obliquely, and perpendicularly to the surface. Mann-Whitney U test. 

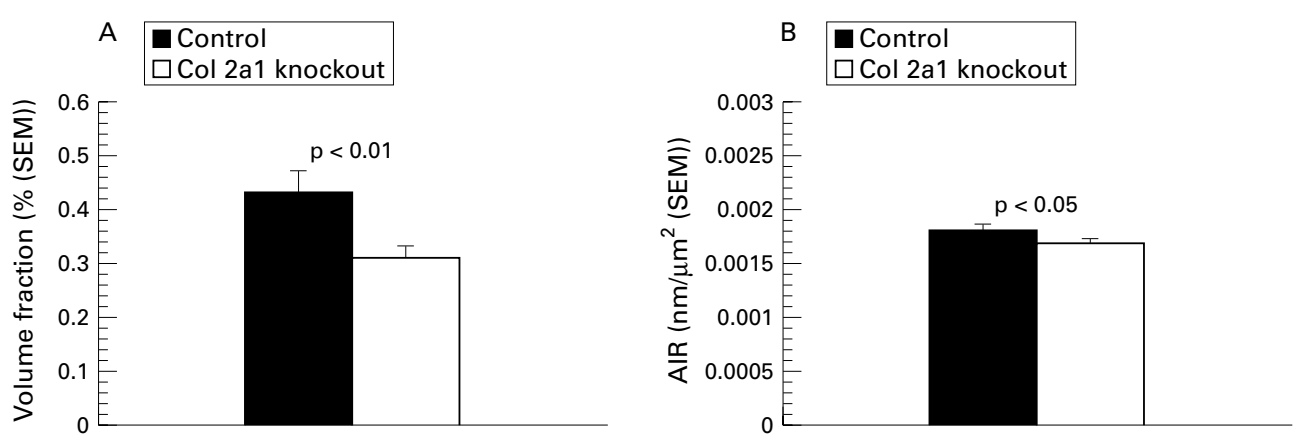

Figure 5 (A) Volume fraction of subchondral bone (mean (SEM)) in the proximal humerus. Mann-Whitney $U$ test. (B) Collagen birefringence in subchondral bone expressed as area integrated retardation (AIR, mean (SEM)) of semicircularly polarised light. Mann-Whitney U test.

superficial and intermediate zones of the knockout mice (fig 4).

The average distance between the osteochondral junction and epiphysial growth plate of humerus did not differ significantly (mean (SEM) 791 (88) $\mu \mathrm{m} v 776(43) \mu \mathrm{m}$ ) between the knockout and wild-type mice, respectively. However, the volume fraction of the subchondral bone, assessed by polarised light, was lower in knockout mice $(31 \%)$ than in controls $(43 \%)(\mathrm{p}<0.01)$ (fig $5 \mathrm{~A})$. In addition, the optical path retardation values for bone matrix collagen were lower in the knockout mice $(\mathrm{p}<0.05)$ (fig 5B).

According to the linear factorial correlation analysis, indentation stiffness was not significantly associated $\left(R^{2} \leqslant 0.3\right)$ with any of the following single parameters: total PG content, total cartilage or zonal thicknesses, birefringence of the cartilage collagen, subchondral bone volume fraction, or subchondral bone birefringence.

\section{Discussion}

Qualitatively, the overall morphology of articular cartilage of the humerus head was normal in the heterozygous Col2a1 knockout mice. The prevalence of superficial OA in the proximal humerus articular cartilage of knockout mice was higher than in the controls $(p=0.002)$. The OA prevalence was lower than found in the knee joint of the same knockout mice. For example, about $85 \%$ of the medial femoral condyles and tibial plateaus of the same animals showed OA changes at the age of 15 months (data not shown). The greater resistance to development of OA in the humerus head compared with the femoral and tibial condyles may be due to a larger and more congruent contact surface in the humeral articulation. Another explanation might be the relatively thick superficial zone of the humeral head (about $35 \%$ of the whole thickness of articular cartilage) - that is, the zone where the main course of collagen fibrils is parallel to the cartilage surface (fig 3A). In the medial tibial plateaus of the same mice the thickness of the superficial zone was only about $10 \%$ (data not shown). Probably, a thick superficial zone with a high degree of fibril parallelism can resist mechanical stress and shear better than a thin superficial zone with a smaller proportion of tangentially oriented collagen fibrils. ${ }^{17}$
In a previous report we carried out a preliminary examination of the same mice. ${ }^{25}$ Inactivation of one of the two alleles of type II procollagen (Col2a1) gene appeared to disturb only slightly the development and properties of murine cartilage and bone tissues, ${ }^{25}$ even though the chondrocytes of newborn mice synthesised only about half the amount of pro$\alpha 1$ (II) chains compared with chondrocytes from wild-type animals. ${ }^{25}$ The situation is analogous with some forms of inheritable chondrodysplasias found in humans where mutations in the Col2al gene lead to reduced synthesis of type II procollagen and assembly of a weaker collagen network predisposing to OA, usually associated with ophthalmopathy and other features of Stickler's syndrome. ${ }^{9}$ The neonatal bone length development also lagged behind in the knockout mice, probably because of minor alterations in the growth plate cartilage. ${ }^{25}$ In addition, the differences between the heterozygous knockout and wild-type mice were less apparent when the mice grew older. ${ }^{25}$ In contrast, chondrocytes of the homozygous knockout mice could not synthesise type II procollagen, and the mice showed severe developmental disturbances of cartilage and bones, especially of the spine. The animals died at birth or earlier during pregnancy. ${ }^{25}{ }^{34} \mathrm{In}$ transgenic mice which expressed a structurally altered pro- $\alpha 1$ (II) chain together with normal chains, the alterations were also severe, but not necessarily lethal. Mild to severe chondrodysplasias are among the syndromes seen in these mice. $^{23} 2434$ The mutated pro- $\alpha 1$ (II) chains are incorporated into the procollagen molecule and thereby destabilise the collagen triple helix. This in turn causes degradation of both abnormal and normal chains of the type II procollagen by a process called "procollagen suicide". ${ }^{24}$ Also, in the transgenic mice there were dilatation of the cisternae of rough endoplasmic reticulum in chondrocytes, reduced amount of collagen fibrils in the matrix, early degeneration of articular cartilage through OA, and disturbed bone growth with decreased collagen and mineral contents. ${ }^{23}{ }^{34}$ Compared with the transgenic mice, which produce structurally altered pro- $\alpha 1$ (II) chains, the heterozygous Col2a1 knockout mice of this study appear to express a phenotype with only minor developmental alterations in cartilage and bone. 
Softening of articular cartilage in heterozygous Col2al knockout mice, as found by the indentation analysis, was probably primarily due to the altered structure and composition of the collagen network in the intermediate and deep zones as shown by PLM. The superficial fibrillation of articular cartilage of heterozygous knockout mice may also contribute to the softening. The fibrillation did not, however, reduce the superficial collagen induced birefringence (fig 3B). This suggests that the superficial collagen network was not injured to a large extent. The indentation test values may also have been influenced by alterations of the subchondral bone-that is, the reduced volume fraction and reduced collagen induced birefringence of the subchondral bone. These changes can be expected to make subchondral bone softer. Earlier, it has been shown that the elastic modulus of human articular cartilage is lower than that of the subchondral, trabecular, or cortical bones. ${ }^{35}$ It is noteworthy that in earlier reports on bone changes in OA, subchondral sclerosis - that is, stiffening and not softening of the subchondral bone, has been reported to take place because of bone mineralisation and osteoid formation. ${ }^{36}$ In this study no signs of subchondral bone sclerosis in humerus were detected. Possibly, despite the minor degenerative and collagen fibril network changes, the cartilage was still mechanically competent and could absorb stresses and strains without significant further damage. The decreased subchondral bone volume fraction might have resulted from the reduced type II collagen expression in the early growth cartilage of humerus, affecting the development of bone that follows. ${ }^{25}$ During limb development, type II collagen is localised throughout the extracellular matrix of the early limb primordium. Later, its distribution shifts towards the developing epiphysial ends and articulating surfaces of the diarthrodial joint. ${ }^{37}$

According to PLM analysis, the elimination of one allele of the Col2al gene did not markedly interfere with the formation of a properly organised collagen network. This conclusion is also supported by the lack of evident disturbances in the zonal and columnar distribution of chondrocytes as well as the qualitatively normal transmission electron microscopic structure of chondrocytes. ${ }^{25}$ It seems that the formation of a functionally appropriate collagen network can take place if the structure and conformation of the pro- $\alpha 1$ (II) chains are normal, though reduced in amount. On the other hand, the homozygous inactivation of the Col2al gene has been shown to cause a severe chondrodysplastic phenotype and perinatal death. ${ }^{25}$ During spine development, the homozygous knockout of type II collagen severely disturbs the removal of the notochord and formation of the intervertebral disc. ${ }^{34}$ The decreased birefringence of collagen fibrils in the heterozygous knockout mice can be explained by a lower collagen content, or fibril orientation, especially in the intermediate and deep zones of articular cartilage. Because we used orientation independent, semicircularly polarised light in our measurements, the area integrated retardation difference probably did not result from an altered fibril direction in the heterozygous knockout mice. The reduction of collagen birefringence in subchondral bone may have a different explanation. The hypothesis can be proposed that the slightly deficient articular and growth cartilages delayed or altered the normal subchondral and trabecular bone development.

In regression analysis there was no significant single correlation between the collagen, the proteoglycan, or the subchondral bone parameters and the indentation stiffness that would have been helpful in the prediction of the biomechanical properties of articular cartilage. This implies that the compressive stiffness of articular cartilage in heterozygous knockout mice, depends on several cartilage parameters and not only on a single one. It should also be noted that indentation stiffness, determined from the equilibrium stress-strain slope, is a structural parameter which is affected by the material properties - that is, elastic modulus and Poisson's ratio of the tissue, as well as by the cartilage thickness. ${ }^{38}{ }^{39}$ Although cartilage thickness was similar in all experimental groups, differences in stiffness values indicate significant alterations of elastic properties of the cartilage matrix. Unfortunately, there is no analytical solution for the biphasic indentation problem when an impervious indenter is used. As a matter of fact, extensive numerical analysis using anisotropic poroelastic ${ }^{40}$ or poroviscoelastic $^{41}$ finite element techniques would be required to solve the problem satisfactorily. The advantage of the more rigorous numerical analysis would also be limited by the assumptions about the theoretically formulated indentation geometry, such as an infinitely wide cartilage layer resting on a totally rigid half space (bone). In the small mouse humerus head, also accompanied by significant changes of the bone structure in the knockout animals, these assumptions cannot be satisfactorily fulfilled. As far as we know, this study is the first successful approach in which values of cartilage stiffness in the mouse bone end have been related to quantitative microscopic estimators. Values from the in situ mechanical testing of humerus head were correlated with the structure of the collagen network and concentration of proteoglycans from the same area.

In conclusion, the inactivation of the Col2a 1 gene made murine articular cartilage softer, altered its collagenous network in the intermediate and deep zones, reduced the subchondral bone volume, and altered its microstructure. Changes in the cartilage collagen network probably contributed to an increased susceptibility to OA.

Support from the Finnish Research Council for Physical Education and Sports, Ministry of Education in Finland; Academy of Finland; Kuopio University Hospital, EVO-grant 5103; grant AR3970 from the US National Institutes of Health is gratefully acknowledged.

\footnotetext{
1 Mendler M, Eich Bender SG, Vaughan L, Winterhalter KH, types II, IX, and XI. J Cell Biol 1989;108:191-7. 2 Eyre DR, Wu JJ. Collagen structure and cartilage matrix Eyre DR, Wu JJ. Collagen structure and cartict
integrity. J Rheumatol Suppl 1995;43:82-5.
} 
3 Prockop DJ, Kivirikko KI. Collagens: molecular biology, diseases, and potentials for therapy. Annu Rev Biochem 1995;64:403-34.

4 Hardingham TE, Fosang AJ. Proteoglycans: many forms and many functions. FASEB J 1992;6:861-70

5 Mow VC, Ratcliffe A, Poole AR. Cartilage and diarthrodial joints as paradigms for hierarchical materials and structures. Biomaterials 1992;13:67-97.

6 Bader DL, Kempson GE. The short-term compressive properties of adult human articular cartilage. Biomed properties of adult human

7 Deak F, Piecha D, Bachrati C, Paulsson M, Kiss I. Primary structure and expression of matrilin-2, the closest relative of cartilage matrix protein within the von Willebrand factor type A-like module superfamily. J Biol Chem 1997;272: 9268-74

8 Broom ND, Silyn Roberts H. Collagen-collagen versus collagen-proteoglycan interactions in the determination of cartilage strength. Arthritis Rheum 1990;33:1512-17.

9 Kuivaniemi H, Tromp G, Prockop DJ. Mutations in fibrillar collagens (types I, II, II, and XI), fibril associated collagen (type IX), and network-forming collagen (type X) cause a
spectrum of diseases of bone, cartilage, and blood vessels. spectrum of diseases of bone,

10 Broom ND, Silyn Roberts H. The three-dimensional 'knit' of collagen fibrils in articular cartilage. Connect Tissue Res 1989;23:261-77.

11 Speer DP, Dahners L. The collagenous architecture of articular cartilage. Correlation of scanning electron microscopy and polarized light microscopy observations. Clin Orthop 1979;139:267-75.

12 Aspden M, Hukins D. Collagen organization in articular cartilage, determined by X-ray diffraction, and its relationship to tissue function. Proc R Soc Lond 1981;212:299-304.

13 Jeffery A, Blunn G, Archer C, Bentley G. Threedimensional collagen architecture in bovine articular cartilage. J Bone Joint Surg Br 1991;73:795-801

14 Hwang WS, Li B, Jin LH, Ngo K, Schachar NS, Hughes GNF. Collagen fibril structure of normal, aging, and osteoarthritic cartilage. J Pathol 1992;167:425-33.

15 Kaab MJ, Ito K, Clark JM, Notzli HP. Deformation of articular cartilage collagen structure under static and cyclic loading. J Orthop Res 1998;16:743-51.

16 Arokoski JP, Hyttinen MM, Lapvetelainen T, Takacs P, Kosztaczky B, Modis L, et al. Decreased birefringence of the superficial zone collagen network in the canine knee the superficial zone collagen network in the canine knee
(stifle) articular cartilage after long distance running train(stifle) articular cartilage after long distance running training, detected by quantitative polaris
Ann Rheum Dis 1996;55:253-64.

17 Arokoski J, Hyttinen M, Helminen H, Jurvelin J Biomechanical and structural characteristics of canine femoral and tibial cartilage. J Biomed Mater Res 1999;48: 99-107.

18 Kaab MJ, Gwynn IA, Notzli HP. Collagen fibre arrangement in the tibial plateau articular cartilage of man and
other mammalian species. J Anat 1998;193(Pt 1):23-34.

19 Hollander AP, Pidoux I, Reiner A, Rorabeck C, Bourne R, Poole AR. Damage to type II collagen in aging and osteoarthritis starts at the articular surface, originates osteoarthritis starts at the articular surface, originates progressive degeneration. J Clin Invest 1995;96:2859-69.

20 Panula HE, Hyttinen MM, Arokoski JP, Langsjo TK, Pelttari A, Kiviranta I, et al. Articular cartilage superficial zone collagen birefringence reduced and cartilage thickness increased before surface fibrillation in experimental osteoarthritis. Ann Rheum Dis 1998;57:237-45.

21 Freeman MA. Is collagen fatigue failure a cause of osteoarthrosis and prosthetic component migration? A hypothesis. J Orthop Res 1999;17:3-8.

22 Vikkula M, Metsaranta M, Ala-Kokko L. Type II collagen mutations in rare and common cartilage diseases. Ann Med $1994 ; 26: 107-14$
23 Metsaranta M, Garofalo S, Decker G, Rintala M, de Crombrugghe $\mathrm{B}$, Vuorio $\mathrm{E}$. Chondrodysplasia in transgenic mice harboring a 15-amino acid deletion in the triple helical harboring a 15-amino acid deletion in the triple helical
domain of pro alpha 1(II) collagen chain. J Cell Biol 1992; domain of pro a

24 Helminen HJ, Kiraly K, Pelttari A, Tammi MI, Vandenberg $\mathrm{P}$, Pereira R, et al. An inbred line of transgenic mice expressing an internally deleted gene for type II procollagen (COL2A1). Young mice have a variable phenotype of a chondrodysplasia and older mice have osteoarthritic changes in joints. J Clin Invest 1993;92:582-95.

25 Li SW, Prockop DJ, Helminen H, Fassler R, Lapvetelainen $\mathrm{T}$, Kiraly $\mathrm{K}$, et al. Transgenic mice with targeted inactivation of the Col2 alpha 1 gene for collagen II develop a skeleton with membranous and periosteal bone but no endochondral bone. Genes Dev 1995;9:2821-30.

26 Busler DE, Li S-W. Rapid screening of transgenic type II and type XI knock-out mice with three-primer PCR. Biotechniques 1996;21:1002-4

27 Hogan B, Constantini F, Lacy E. Manipulating the mouse embryo. A laboratory manual. New York: Cold Spring embryo. A laboratory manual.

28 Jurvelin JS, Buschmann MD, Hunziker EB. Optical and mechanical determination of Poisson's ratio of adult bovin humeral articular cartilage. J Biomech 1997;30:235-41.

29 Hoch DH, Grodzinsky AJ, Koob TJ, Albert ML, Eyre DR. Early changes in material properties of rabbit articular cartilage after meniscectomy. J Orthop Res 1983;1:4-12.

30 Jurvelin JS, Rasanen T, Kolmonen P, Lyyra T. Comparison of optical, needle probe and ultrasonic techniques for the measurement of articular cartilage thickness. J Biomech 1995;28:231-5.

31 Kiraly K, Hyttinen MM, Lapvetelainen T, Elo M, Kiviranta $\mathrm{I}$, Dobai J, et al. Specimen preparation and quantification of collagen birefringence in unstained sections of articular cartilage using image analysis and polarizing light microscopy. Histochem J 1997;29:317-27.

32 Kiviranta I, Jurvelin J, Tammi M, Säämänen A-M, Helminen $H$. Microspectrophotometric quantitation of glycosaminoglycans in articular cartilage sections stained with safranin O. Histochemistry 1985;82:249-55.

33 Lapvetelainen T, Nevalainen T, Parkkinen JJ, Arokoski J, Kiraly $\mathrm{K}$, Hyttinen $\mathrm{M}$, et al. Lifelong moderate running training increases the incidence and severity of osteoarthritis in the knee joint of C57BL mice. Anat Rec $1995 ; 242: 159-65$

34 Aszodi A, Chan D, Hunziker E, Bateman JF, Fassler R. Collagen II is essential for the removal of the notochord and the formation of intervertebral discs. J Cell Biol 1998;143: $1399-412$.

35 Choi K, Kuhn JL, Ciarelli MJ, Goldstein SA. The elastic moduli of human subchondral, trabecular, and cortical bone tissue and the size-dependency of cortical bone modulus. J Biomech 1990;23:1103-13.

36 Grynpas MD, Alpert B, Katz I, Lieberman I, Pritzker KP. Subchondral bone in osteoarthritis. Calcif Tissue Int 1991; 49:20-6.

37 Morrison EH, Ferguson MWJ, Bayliss MT, Archer CW. The development of articular cartilage: I. The spatial and
temporal patterns of collagen types. J Anat 1996;189:9-22.

38 Hayes WC, Keer LM, Herrmann G, Mockros LF. A mathematical analysis for indentation tests of articular cartilage. J Biomech 1972;5:541-51

39 Mak AF, Lai WM, Mow VC. Biphasic indentation of articuar cartilage: I. Theoretical analysis. J Biomech 1987;20: 703-14.

40 Mow VC, Grood PM, Gardner TR. A new method to determine the tensile properties of articular cartilage using the indentation test. Transactions of the 46th Annual Meeting of the Orthopaedic Research Society 2000;25:103.

41 Suh JK, Bai S. Biphasic poroviscoelastic behavior of articular cartilage in creep indentation test. Transactions of the 43rd Annual Meeting of the Orthopaedic Research Society 1997;22:823. 